\title{
OBTAINING OF THE IN VITRO Solanum tuberosum CALLUSES WITH HIGH $\alpha$-SOLANINE CONTENT IN THE CELLS THAT MANIFEST ANTIMICROBIAL ACTIVITY
}

\author{
Kuznetsova Elena, Fomicheva Anna, Kuznetsova Elena, Stelmashchuk Olga, Seregina Evgenia, Dunaev A. \\ Oryol State University named after I.S. Turgenev, Russia \\ e-mail: elkuznetcova@yandex.ru
}

To combat diseases and insects in agricultural production, synthetic drugs are widely used. However, the use of many known pesticides has a negative impact on the environment. As a result, the search for producers of biological agents with a fungicidal and insecticidal action continues.

A number of researchers have found that $\alpha$-solanine has a fungicidal effect. As a producer of glycoalcaloids, especially $\alpha$-solanine it Solanum tuberosum has attracted the attention of researchers. In vitro cell cultures, which allow the production of synthetic products of secondary metabolites throughout the year and in a more concentrated form compared to a whole plant, are of particular interest.

Callus culture of Solanum tuberosum was performed by the solid phase method on our Murasige-Skoog modified medium at a temperature of $26{ }^{\circ} \mathrm{C}$, air humidity of $60-70 \%$ and illumination of 1000 lux. The results of the study show that the modification of the nutrient medium by the addition of tryptophan and cholesterol contributed to the acceleration of the regeneration of the callus culture. Using a ZeissDiscovery V20 microscope, we studied the morphology of callus cells, which showed that the modification of the nutrient medium increases the number of cells, the cells are smaller, but the number of chloroplasts increases. noticeably.

With an increase in the growth index, a specific growth rate of $25-29 \%$ and a decrease in biomass doubling time of 22-28\%, solanine yield increases.

A rapid method for the determination of solanine in Solanum tuberosum callus by fluorescence spectroscopy was developed. By evaluating the fluorescence contrast coefficient, we can evaluate the contribution of various fluorophores to the spectrum of the total signal. The spectrum of solanine was detected at a wavelength of $530 \mathrm{~nm}$. The characteristic peak of the glycoside in the spectrogram can be used to quantify it.

On the basis of the callus culture, planting material containing up to $0.56 \%$ solanine was obtained, calculated on a perfectly dry mass of raw material resistant to fungal infections caused by fungi of the genus Fusarium.

Solanin powder was obtained from the solanum tuberosum callus biomass by extraction with $5 \%$ acetic acid, followed by precipitation with $25 \%$ ammonia solution, cooling and drying. extraction with $95 \%$ ethanol, which was then evaporated to dryness. Experiments on pure cultures of microorganisms have shown that the use of solanine powder as a fungicidal preparation inhibits fungi Aspergillus candida, Penicillium expansion, Mucor mucedo, Rhizopus stolonifer, certain species of Fusarium and bacteria of the species Bacillus subtilis.

Thus, a technology has been developed for the production of Solanum tuberosum callus biomass containing an increased amount of solanine. On the basis of callus culture, a seed resistant to certain fungal infections and a fungicidal solanine preparation was obtained. A method for rapid diagnosis of solanine in plant material has also been developed. 